\title{
Characterization of Exercise-Induced Cytokine Release, the Impacts on the Body, the Mechanisms and Modulations
}

\author{
Katsuhiko Suzuki* \\ Faculty of Sport Sciences, Waseda University, Japan
}

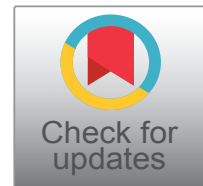

*Corresponding author: Katsuhiko Suzuki, MD \& PhD, Professor, Faculty of Sport Sciences, Waseda University, 2-579-15 Mikajima, Tokorozawa, Saitama 359-1192, Japan, ORCID iD: 0000-0002-6572-5809

\begin{abstract}
The objective of this review is to introduce significant research findings mainly on cytokine responses to exercise. First, some basic background information on cytokines is provided. Then, some of our data according to exercise modes and key experimental research on the factors affecting cytokine responses to exercise are explained. Furthermore, the mechanisms and modulations of the cytokine responses are described to understand stress and inflammatory reactions and their prevention/recovery.
\end{abstract}

\section{Keywords}

Exhaustion, Cytokine, Chemokine, Systemic inflammation, Muscle damage, Anti-inflammatory effect of exercise

\section{Introduction}

Cytokines are important intercellular signaling molecules that regulate inflammation and immune responses [1]. They are produced by a variety of cells and are classified into several different categories based on their bioactivity. Physiologically, cytokines act locally in an autocrine or paracrine manner at very low concentrations. These actions are in contrast to hormones, which exert their influence through the systemic circulation on target organs. However, pathologically, cytokines are released systemically in response to a variety of serious insults such as systemic infections, severe trauma, burn injury, and acute myocardial infarction. Therefore, the increase in the circulating concentration of cytokines (hypercytokinemia) is considered abnormal and is collectively designated as a Systemic Inflammatory Response Syndrome (SIRS) clinically [2-4].
As for the classification of cytokines [1,2], there are pro-inflammatory cytokines such as tumor necrosis factor (TNF)- $\alpha$, interleukin (IL)-1 $\beta$ and IL- 6 , which induce acute inflammation. Immunomodulatory cytokines such as IL-2, interferon (IFN)- $\gamma$ and IL-12p70 are produced to induce cellular immunity as protection against pathogens such as viruses and intracellular bacteria. Figure 1 shows the cytokine balance based on helper $\mathrm{T}$ (Th) cells, and the effects of exhaustive exercise on the component cytokines are described later (Figure 1) [5]. There are also anti-inflammatory cytokines such as IL-1 receptor antagonist (IL-1ra), IL-4, IL-6, IL10 and IL-12p40, which block action and production of other pro-inflammatory and immunomodulatory cytokines. Here, IL-6 is classified as a multi-functional cytokine, because it can act as both a pro- and an antiinflammatory cytokine, depending on the progression of inflammation. Colony-stimulating factors are involved in hematopoiesis, mobilization and activation of neutrophils and monocytes. Chemokines are chemotactic cytokines that regulate tissue infiltration of leukocytes.

This article firstly introduces findings on the effects of most exhaustive exercise conditions, a maximal intensity exercise within $30 \mathrm{~min}$, and a long duration race such as marathon, duathlon and triathlon on cytokine release in observational studies. Then, some of the main experimental research concerning the impacts on the body and the underlying mechanisms of endurance exercise-related phenomena are highlighted. Besides, our findings of cytokine dynamics following eccentric exercise in relation with muscle damage were paradox-

Citation: Suzuki K (2019) Characterization of Exercise-Induced Cytokine Release, the Impacts on the Body, the Mechanisms and Modulations. Int J Sports Exerc Med 5:122. doi.org/10.23937/24695718/1510122

Accepted: March 26, 2019; Published: March 28, 2019

Copyright: (C) 2019 Suzuki K. This is an open-access article distributed under the terms of the Creative Commons Attribution License, which permits unrestricted use, distribution, and reproduction in any medium, provided the original author and source are credited. 


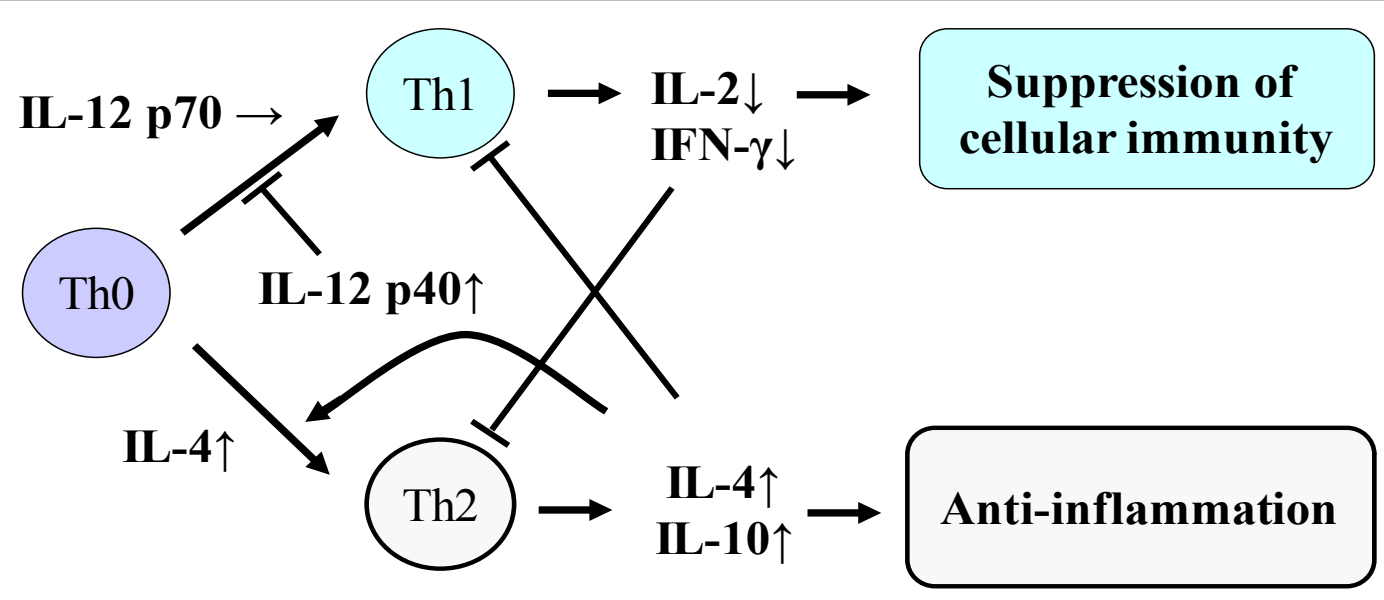

Figure 1: Cytokine balance based on helper T (Th) cells. Suzuki K, et al. [2,5].

ically strengthened and the prevention/modifications are described. Past comprehensive and related reviews on cytokine response to exercise are referred to elsewhere $[2,5-10]$.

\section{Effects of Short Maximal Exercise on Cytokine Response}

In the mid-1990s, we had investigated the effects of exercise on immune perturbations such as neutrophil mobilization and priming following exhaustive exercise and the underlying mechanisms [11-13]. As for cytokines, at first, we measured serum concentrations of TNF- $\alpha, I L-1 \beta$ and IFN- $\gamma$ before and after maximal exercise on a treadmill and $1 \mathrm{~h}$ after exercise [13]. Although we observed exercise-elicited leukocytosis due to increases in large granular lymphocytes, monocytes and neutrophils together with enhanced neutrophil pseudopod projection and the capacity to produce Reactive Oxygen Species (ROS) [11], we could not detect the above cytokines at least up to $1 \mathrm{~h}$ after exercise [13]. It might be partly because the sensitivity of the Enzyme-Linked Immunosorbent Assays (ELISA) was low at that time, and it was later found that these cytokines are not easily increased in the circulation after short-time maximal exercise as compared to long endurance exercise $[2,5,6,10]$.

In spite of the disappointed results even using expensive ELISA kits, we tried once again to investigate the time course of changes until $2 \mathrm{~h}$ after maximal exercise in a broader spectrum of cytokines in plasma and urine as well as employing more sensitive ELISA [14]. As a result, we found that plasma IL-1 $\beta$ was significantly elevated $2 \mathrm{~h}$ after exercise by two-fold. In urine, IL-1ra increased 15.5 fold $1 \mathrm{~h}$ after exercise, and TNF- $\alpha 5$ fold $2 \mathrm{~h}$ after exercise. Plasma and urine IL-4 levels were significantly elevated in parallel only 2 h after exercise by 7 fold. Plasma Granulocyte ColonyStimulating Factor (G-CSF) was significantly elevated immediately after exercise, which remained until 2 $\mathrm{h}$ after exercise. Granulocyte Macrophage ColonyStimulating Factor (GM-CSF) in plasma was temporarily elevated immediately after exercise, and the urine level was also elevated $1 \mathrm{~h}$ later, indicating a temporal secretion into the circulation and subsequent excretion into urine.

These data suggested that some cytokines were rapidly excreted from the circulation and that cytokine in plasma might only reflect a minor portion of the entire amount produced in the body. Also, it was suggested that exercise stress promoted the release of cytokine antagonist and anti-inflammatory cytokines to prevent systemic inflammation. Additionally, early increments of G-CSF and GM-CSF suggest the involvement of mobilization and priming of leukocytes, which were partly demonstrated later [2,15-17]. These changes indicated that each cytokine dynamics and impacts on the body might be quite different $[2,6,9,10]$.

\section{Effects of Long Endurance Races on Cytokine Response}

Our original paper submission on marathon data was rejected again and again, and it took several years to be published finally in 2000 [18]; it was certain that the results were no changes in plasma pro-inflammatory cytokines even after a competitive full marathon race, and immunomodulatory cytokine IL-2 decreased after exercise. However, anti-inflammatory cytokines such as IL-1ra, IL-6 and IL-10 increased dramatically after the race. These changes might result in immunosuppression after exhaustive exercise $[2,5,9,18]$. On the other hand, colony-stimulating factors and chemokines increase significantly, which might induce mobilization and activation of neutrophils and monocytes, resulting in inflammation $[18,19]$.

Particularly, IL-6 increased more than 100 fold following a marathon race and was correlated with changes in IL-10 $[2,7,18,19]$, the most potent immunosuppressive cytokine. IL-6 was also correlated with neutrophil count, whereas neutrophil mobilization was correlated with changes in the muscle damage markers, Creatine Kinase (CK) and myoglobin [20]. Interestingly, IL-6 response was also correlated with 


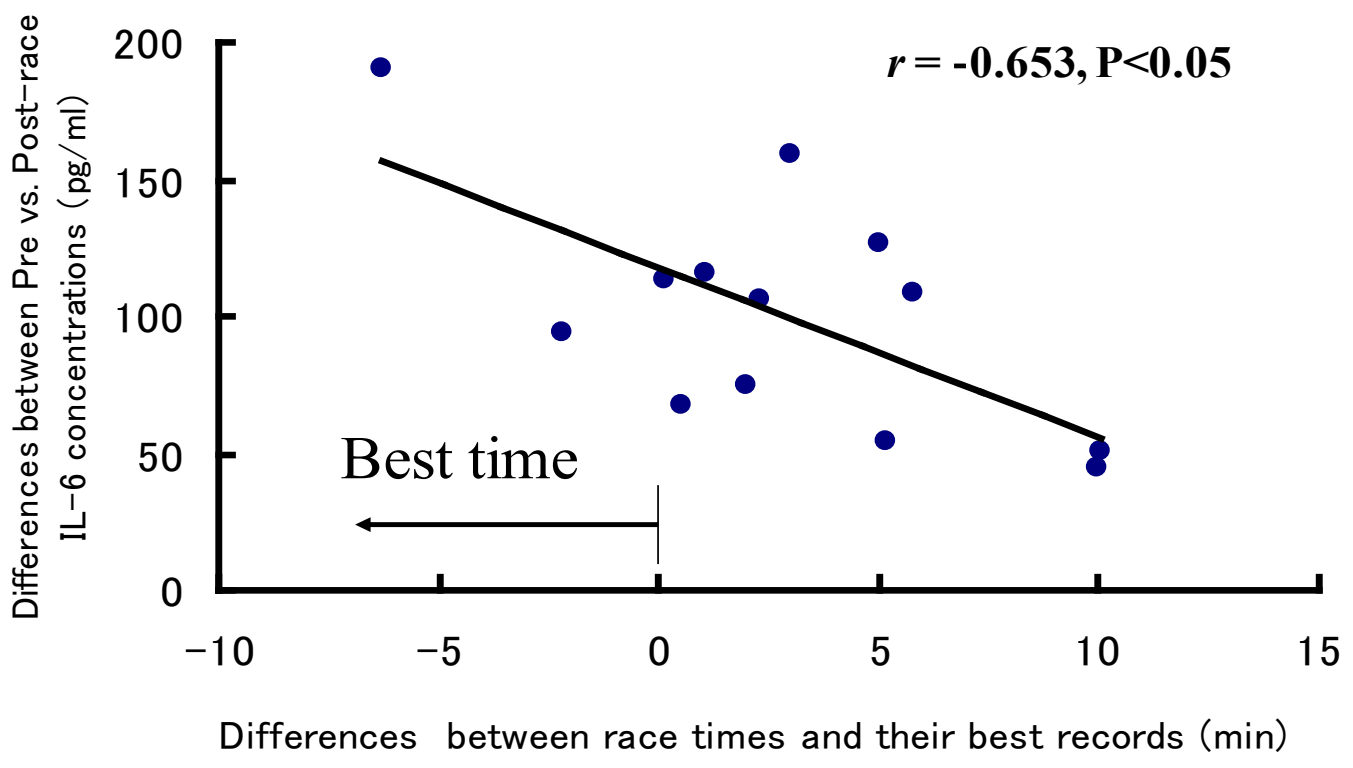

Figure 2: IL-6 response and marathon race time. Suzuki K, et al. [5].

increases in free fatty acids [5], which are an important energy substrate during endurance exercise. Another interesting observation from our research was that IL-6 responses were inversely related to individual race time (Figure 2) [5]. That is, IL-6 was highest in those athletes who run fastest. This observation might be related to the effects of IL- 6 on the mobilization of free fatty acids [21-23]. Although IL-6 has been classified as a multifunctional cytokine, it might perform several different roles during exercise. Also, anti-inflammatory effects of IL-10 might be induced for suppression of ROS production by neutrophils and monocytes [19]. Furthermore, IL-1ra and IL-12p40 might work as antagonists of exerciseinduced inflammation $[2,5]$.

Concerning triathlon and duathlon races [2428], muscle damage and inflammation were more pronounced, and acute kidney injury was also observed $[24,27]$. On the other hand, time course changes in inflammatory markers were examined a longer duration race and the recovery and revealed that the triathlete recovered almost completely within approximately one week using inflammatory markers [28]. However, typical inflammatory markers such as C-Reactive Protein (CRP) were not sensitive enough in case of exercise, but some cytokine responses to exercise were more exaggerated in urine $[2,26]$. As such, experimental research was needed for further characterization of each cytokine dynamics and their roles in the exercise-induced organ damage and inflammation.

\section{Experimental Research on Cytokine Response to Endurance Exercise}

At first, we have extended the study on repeated bouts effects of exercise in relation with hormonal changes to analyze adaptation mechanisms regulating systemic inflammatory responses of the stressed body [20]; endurance exercise induced peripheral neutrophilia and neutrophil priming for ROS production. Plasma IL-6 rose significantly and was closely correlated with the neutrophil responses, which were also correlated with muscle damage markers. These exercise-induced responses were strongest on the first day, but the magnitude gradually decreased with progressive daily exercise, whereas catecholamine responses to exercise sessions gradually rose, possibly suppressing neutrophil priming and may affect local tissue damage of susceptible organs $[15,16,20]$.

Professor Pedersen's research group in Copenhagen, Denmark, has demonstrated that contracting muscle produces IL- 6 and releases it into the circulation depending on the energy demand, and IL- 6 mobilizes energy substrates in a similar manner to stress hormones [29]. Therefore, IL-6 is considered to support energy supply and endurance performance and is therefore referred to as a myokine [7]. However, it was unclear whether IL- 6 is released into the circulation in response to exercise-induced muscle damage or exercise intensity $[2,7,20,29]$.

Dr. Peake from the University of Queensland in Brisbane, Australia, investigated the effects of exercise intensity and muscle damage on neutrophil activities [30]. I assisted in his work by measuring changes in several cytokines that regulate neutrophil activity during exercise [30-32]. He compared three exercise conditions using 10 male endurance-trained athletes. The first condition was moderate-intensity, level treadmill running at $60 \% \mathrm{VO}_{2}$ max for 60 minutes, the second conditions was high-intensity, level treadmill running at $85 \% \mathrm{VO}_{2}$ max for 60 minutes [30,31], and the last was moderate-intensity downhill running at $60 \% \mathrm{VO}_{2}$ max for 60 min [32]. Figure 3 shows the changes in myoglobin, a muscle damage marker. Downhill running caused marked increase in myoglobin, indicating that this protocol caused more muscle damage than moderate- 


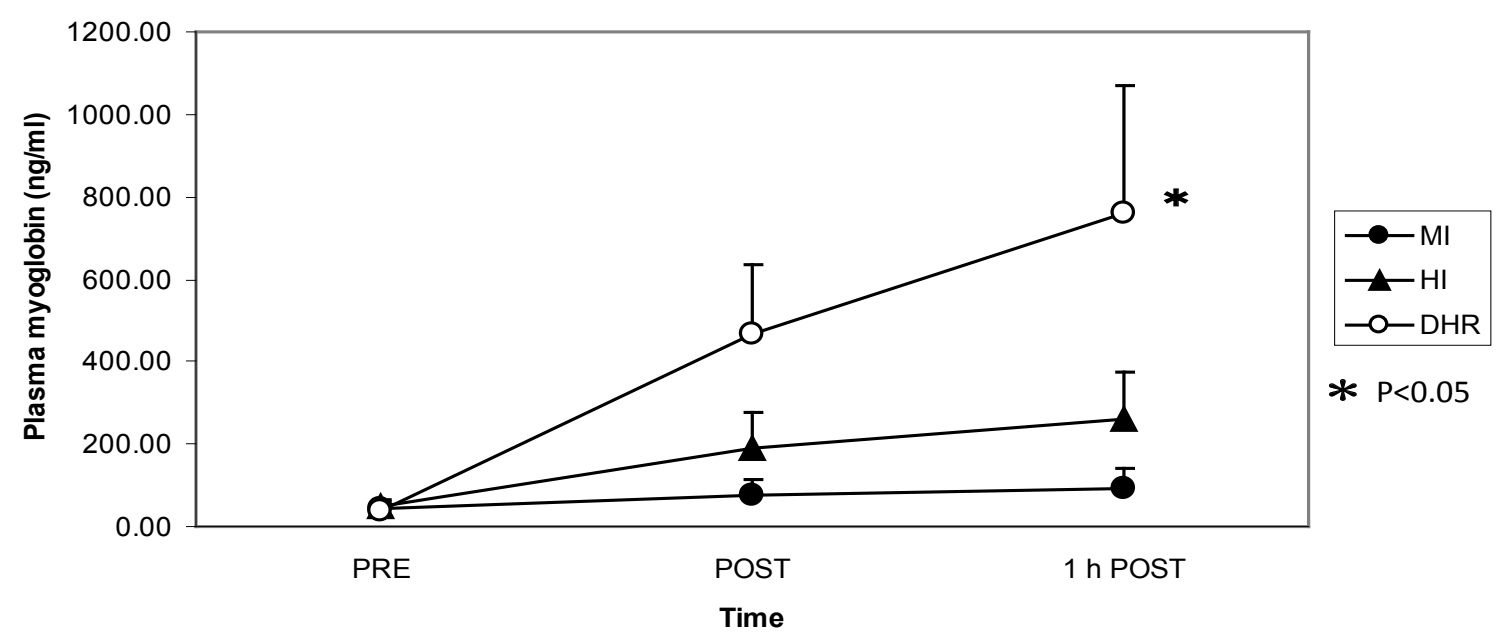

Figure 3: Myoglobin increased after downhill running (DHR) compared with moderate-intensity (MI) and high-intensity (HI) running. Peake J, et al. [30,31].

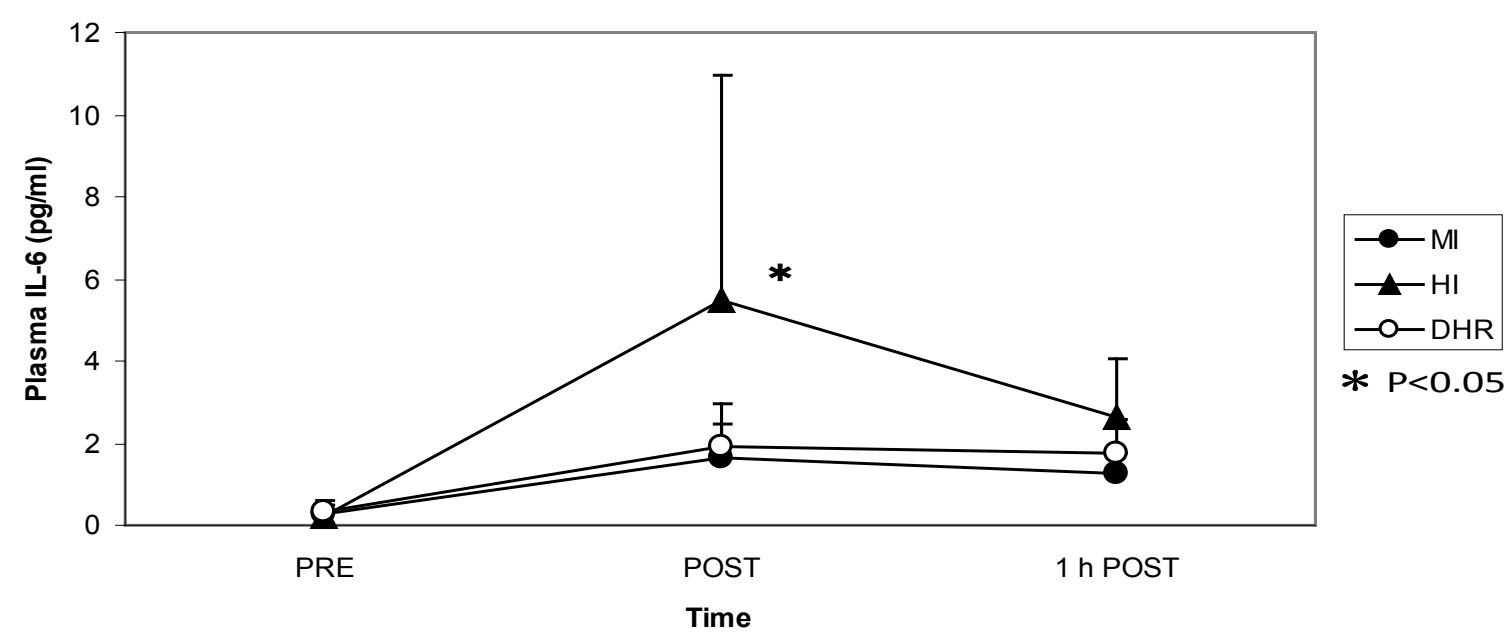

Figure 4: IL-6 increased more during high-intensity $(\mathrm{HI})$ running than during moderate-intensity (MI) and downhill running (DHR). Peake J, et al. [30,31].

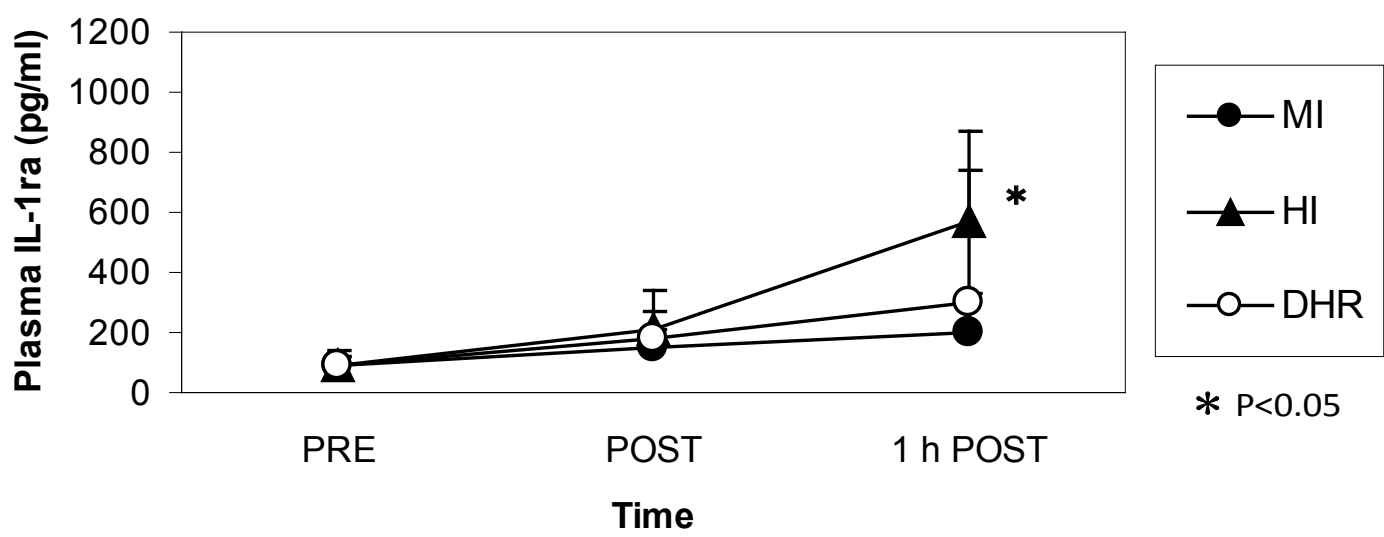

Figure 5: IL-1 ra increased after high-intensity $(\mathrm{HI})$ running but not after moderate-intensity (MI) and downhill running (DHR). Peake J, et al. [30,31].

and high-intensity level running (Figure 3). However, IL-6 response was greater after high-intensity level running compared with moderate-intensity level running and downhill running, indicating that IL- 6 response depends on exercise intensity rather than muscle damage (Figure
4). Also, IL-1ra increased significantly after high-intensity running, but not after downhill running (Figure 5). IL-10 also increased only after high-intensity running (Figure 6). These results suggest that systemic release of IL-6, IL-1ra and IL-10 depends more on exercise intensity 


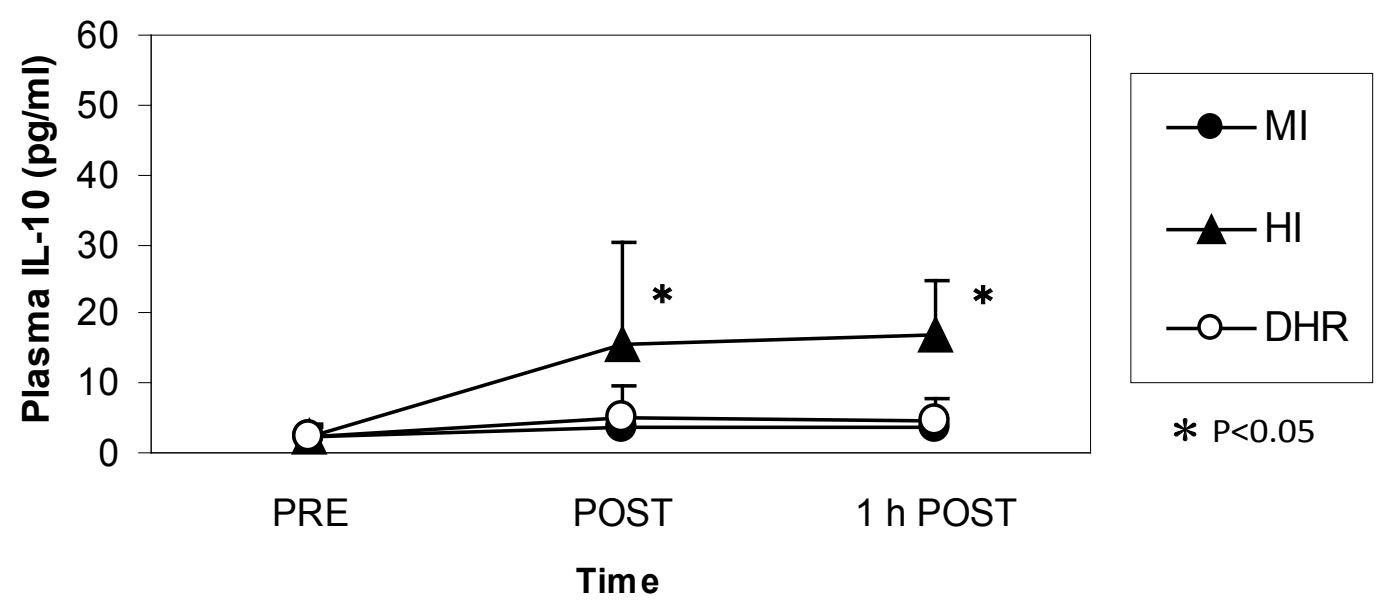

Figure 6: IL-10 increased during high-intensity $(\mathrm{HI})$ running but not during moderate-intensity (MI) and downhill running (DHR). Peake J, et al. [30,31].
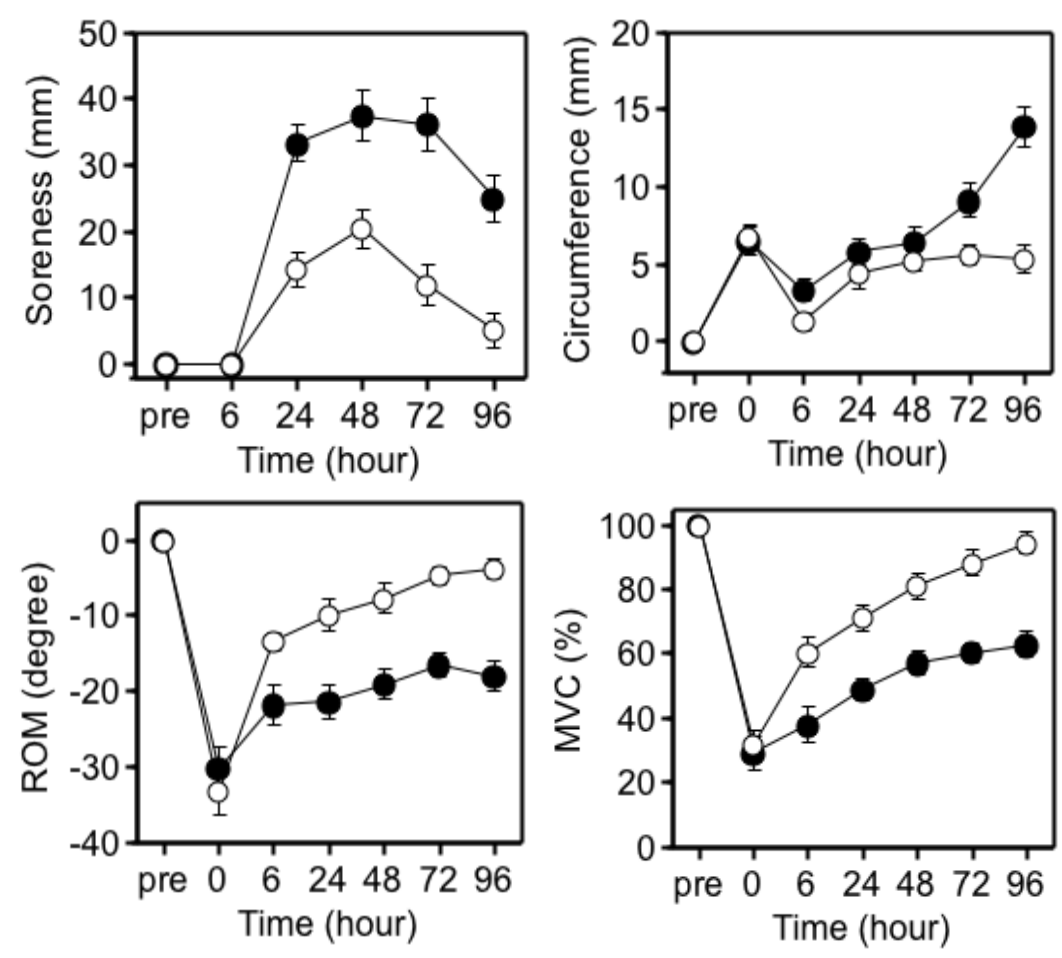

Figure 7: Responses of the elbow flexors to the $1^{\text {st }}(\bullet)$ versus $2^{\text {nd }}(\circ)$ bouts (4 weeks apart) [33]. ROM: range of motion; MVC: maximal voluntary contraction.

than muscle damage [2,8-10]. Thus, it was considered that cytokine responses reflected stress reactions of the body.

\section{Effects of Eccentric Exercise on Cytokine Re- sponse}

Although it was initially proposed that IL-6 is released into the circulation in response to exercise-induced muscle damage, there was little definite evidence to support this concept. To further investigate whether there is a relationship between muscle damage and cytokines, we examined the effects of eccentric exercise which causes local muscle damage, but minimal metabolic stress with the joint study of Professor Nosaka's research group in Perth, Australia [33-35]. Ten untrained male university student repeated eccentric lengthening contraction of the elbow flexors to induce muscle damage and inflammation. Using a dumbbell, eccentric lengthening contractions were performed, and the same protocol was repeated one month later as the second bout to explore the mechanisms of the muscle adaptation. We measured muscle damage markers, cytokines in plasma and urine, and related markers of inflammation. In response to the first bout, inflammatory signs such as muscle pain and swelling, and decreases in range of motion and muscle strength occurred (Figure 7). Muscle damage markers, creatine kinase and myoglobin, also increased dramatically after the first bout (Figure 8). In contrast, these changes were much smaller after the second bout, which indicates 
that muscle adaptation occurred between the two bouts of exercise. In contrast with these marked changes in markers of muscle damage, there were only minor changes in plasma inflammatory mediators [33-35].

We measured renal damage markers and urine cytokine levels as well to check the possibility that plasma cytokines are excreted rapidly from the kidneys into urine. However, no significant differences were observed in renal damage markers in spite of severe muscle damage like rhabdomyolysis, and renal clearance was not affected by the local eccentric exercise and muscle damage. Interestingly, we observed that there were significantly higher and more rapid increases in anti-inflammatory cytokines such as IL-1ra and IL-6 in the second bout compared with the first bout (Figure 9). In summary, although there was severe muscle damage after the eccentric exercise, the changes in cytokines were quite minor, and considerably smaller than that following endurance exercise. Nevertheless, there was induction of anti-inflammatory cytokines in the second bout, which might help restrict muscle damage.

We had also evidence that plasma and urine cytokine levels did not change following local eccentric exercise using a calf raise model as well [36-38]. Even though we discovered a novel marker of muscle damage in urine, $\mathrm{N}$-terminal fragment of titin [38], cytokine changes were not detected even using shotgun proteomics (Figure 10). These findings indicated that muscle damage is not the trigger of the systemic cytokine release.

\section{Effects of Energy Supplementation on Cytokine Response to Exercise}

Energy supply during exercise is important for the endurance performance. In a previous study, Professor Nieman, at Appalachian State University, USA, investigated the effects of carbohydrate drink on plasma cytokine levels and muscle cytokine gene expression following $3 \mathrm{~h}$ of treadmill running at $70 \% \mathrm{VO}_{2}$ max [39]. They demonstrated that carbohydrate drink intake reduced plasma IL- 6 concentrations and muscle IL-6 gene expression. Plasma IL-1ra and IL-10 were also reduced by carbohydrate drink. These findings suggest that carbohydrate ingestion before and during exercise is useful to prevent not only a decline in endurance performance by sparing muscle glycogen stores, but also exercise-induced muscle damage.

However, some studies reported that carbohydrate ingestion after eccentric exercise enhanced the increase in circulating IL-6 levels, gene expression of IL- 8 and MCP1 in skeletal muscle, and muscle soreness [40]. Although carbohydrate ingestion after endurance exercise is recommended to enhance muscle glycogen synthesis,
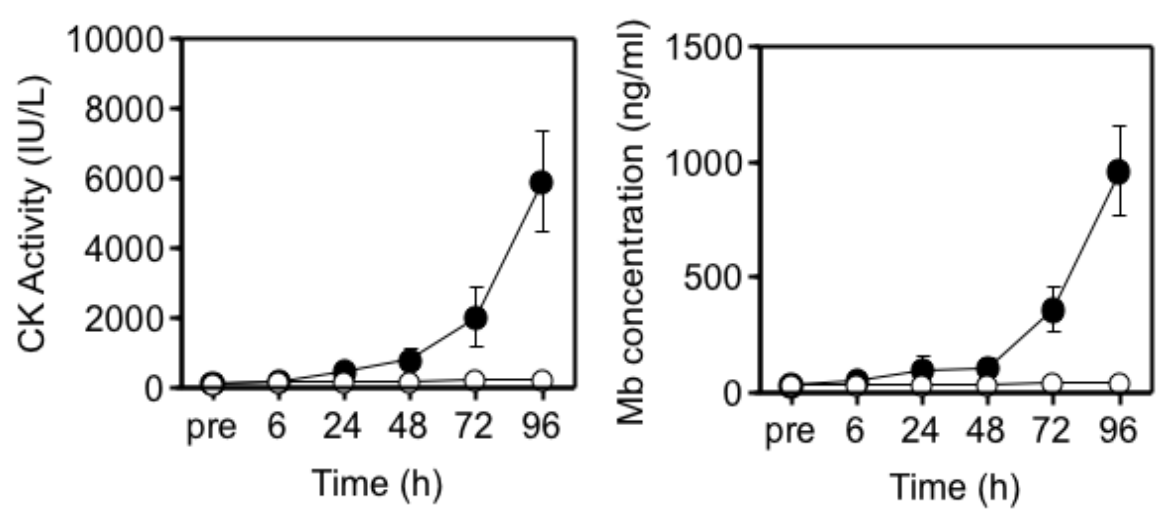

Figure 8: Responses of blood parameters to the $1^{\text {st }}(\bullet)$ versus $2^{\text {nd }}(\circ)$ bouts (4 weeks apart) [33]. $\mathrm{CK}$ : creatine kinase; Mb: myoglobin.

Urinary IL-1ra concentration (corrected for creatinine)

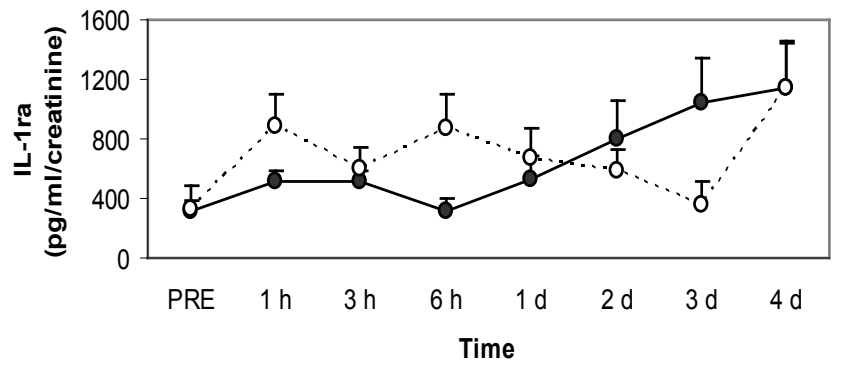

Urinary IL-6 concentration (corrected for creatinine)

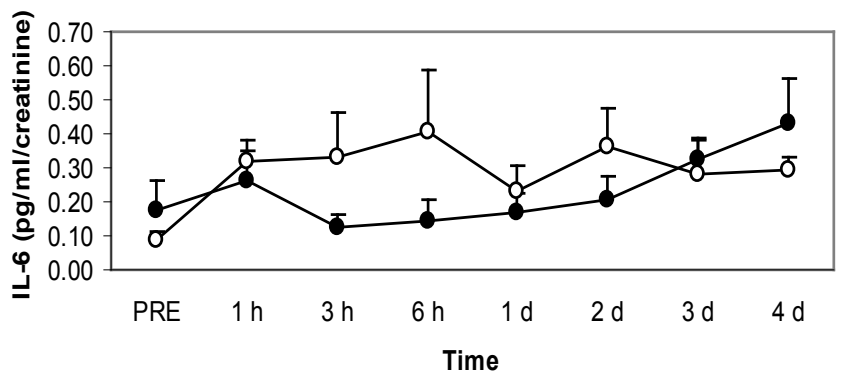

Figure 9: Responses of urinary cytokines to the $1^{\text {st }}(\bullet)$ versus $2^{\text {nd }}(\circ)$ bouts (4 weeks apart) [33]. IL-1ra: interleukin 1 receptor antagonist; IL-6: interleukin 6. 


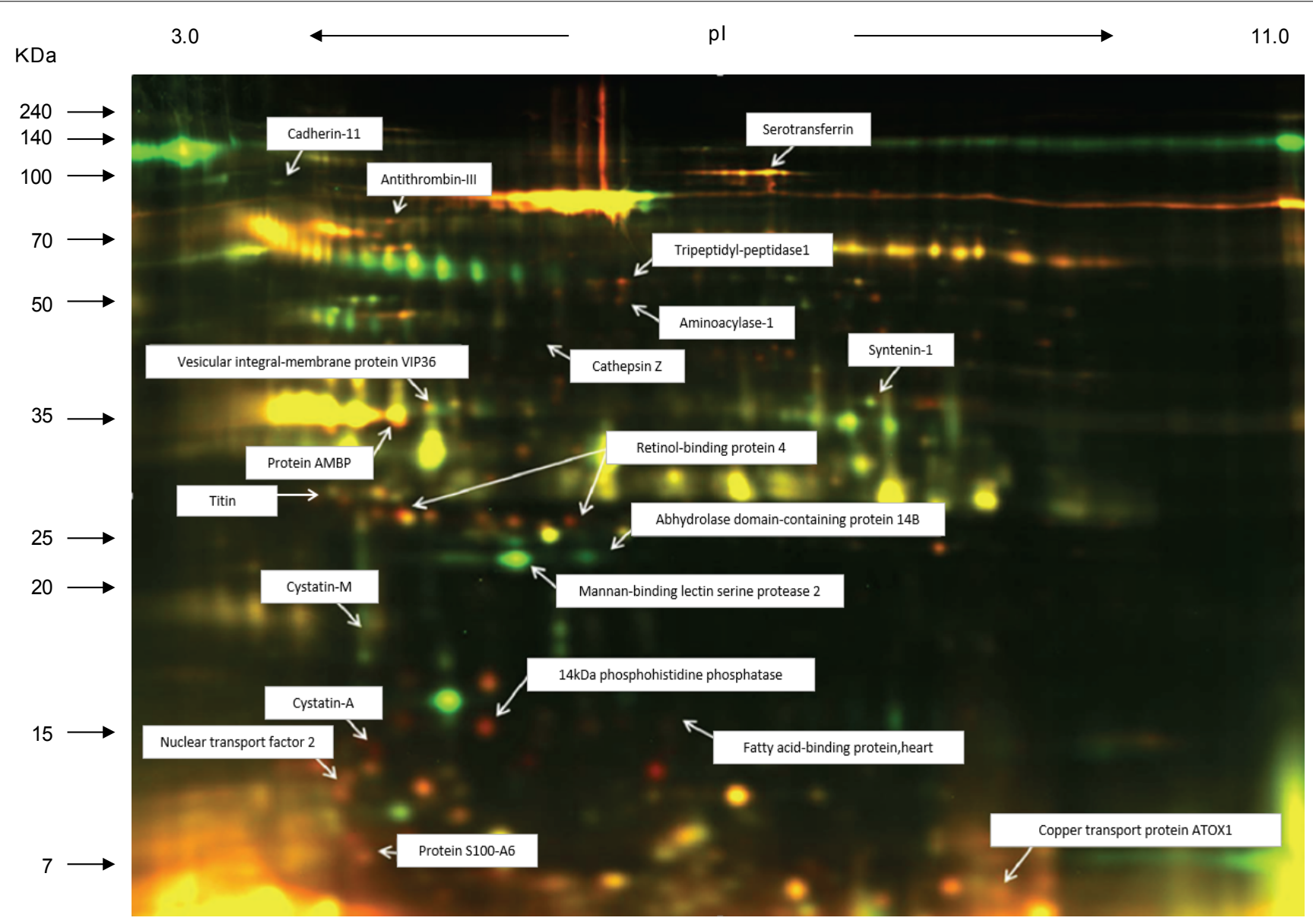

Figure 10: Shotgun proteomics using urine after eccentric exercise [38].

these findings suggest that post-exercise carbohydrate ingestion may promote muscle inflammation via the promotion of neutrophil and macrophage infiltration in skeletal muscle.

We examined the effects of ingestion of different amounts of carbohydrate after endurance exercise on circulating cytokine levels, neutrophil count, and the markers of neutrophil activation and muscle damage, and revealed that carbohydrate ingestion after endurance exercise did not enhance the exerciseinduced increase in above variables, regardless of the amount of carbohydrate ingested [41]. Furthermore, the ingestion of a high amount of carbohydrate maintained high plasma glucose and insulin concentrations during the recovery phase when compared to the ingestion of a low amount of carbohydrate. These findings suggest that the ingestion of a high amount of carbohydrate after endurance exercise provides a favorable condition for the recovery from endurance exercise without an increase in inflammatory responses and markers of muscle damage.

\section{Effects of Environmental Temperature on Cytokine Response to Exercise}

Heat stress might attenuate the effects of carbohydrate on immunoendocrine responses to exercise by increasing endogenous glucose production and reduc- ing the rate of exogenous carbohydrate oxidation. We compared the efficacy of carbohydrate consumption on immune responses to exercise in temperate vs. hot conditions. Ten male cyclists exercised on 2 separate occasions in temperate $\left(18.1 \pm 0.4{ }^{\circ} \mathrm{C}, 58 \% \pm 8 \%\right.$ relative humidity) and on another 2 occasions in hot conditions $\left(32.2 \pm 0.7^{\circ} \mathrm{C}, 55 \% \pm 2 \%\right.$ relative humidity). On each occasion, the cyclists exercised in a fed state for $90 \mathrm{~min}$ at $~ 60 \% \mathrm{VO}_{2}$ max and then completed a $16.1-\mathrm{km}$ time trial. Every 15 min during the first 90 min of exercise, they consumed $0.24 \mathrm{~g} / \mathrm{kg}$ body mass of a carbohydrate or placebo gel. Neutrophil counts increased during exercise in all trials and were significantly lower after the carbohydrate than after the placebo trials in $32^{\circ} \mathrm{C}$. The circulating IL-6, IL-8, IL-10, G-CSF, myeloperoxidase and calprotectin also increased during exercise in all trials but did not differ significantly between the carbohydrate and placebo trials. Carbohydrate ingestion attenuated neutrophil counts during exercise in hot conditions, whereas it had no effect on any other immune variables in either temperate or hot conditions $[42,43]$.

Also, we conducted a study on cytokine responses to exercise in warm and cool environments in a joint study with Professor Kim at Keimyung University in Korea $[44,45]$. The aims of this study was to investigate whether a cool environment affects cytokine responses to exercise. Since skating is very popular in Korea, short- 
track skaters who are adapted to exercise in the cold and inline skaters who are adapted to exercise in warm conditions were recruited for the study. Both groups of athletes exercised in either cool or warm conditions on separate days. In the first protocol, inline skaters and short-track skaters stayed at room temperature for $60 \mathrm{~min}$ before cycling at $65 \% \mathrm{VO}_{2}$ max for $60 \mathrm{~min}$, and then recovered for $2 \mathrm{~h}$. Under these conditions, the short-track skaters who were adapted to exercising in cool conditions were exposed to unaccustomed stress. In the second protocol, the two groups of subjects stayed in a cool environment for 60 min before cycling at $65 \% \mathrm{VO}_{2}$ max for $60 \mathrm{~min}$ in the cool environment, and then recovered at room temperature for $2 \mathrm{~h}$. Under these conditions, the inline skaters who are adapted to exercising in warm conditions were exposed to unaccustomed stress. Among 4 conditions, when the short-track skaters exercised in unaccustomed warm conditions (SW), IL-6 increased dramatically, whereas for these athletes, this response was reduced when exercising in familiar cool conditions (Figure 11). IL-1ra (Figure 12) and IL-10 (Figure 13) also shows the highest response in SW. Cortisol was elevated immediately after exercise, but the most dramatic changes were observed in SW (Figure 14). Cortisol responses were smaller following exercise in the cool in both groups. As
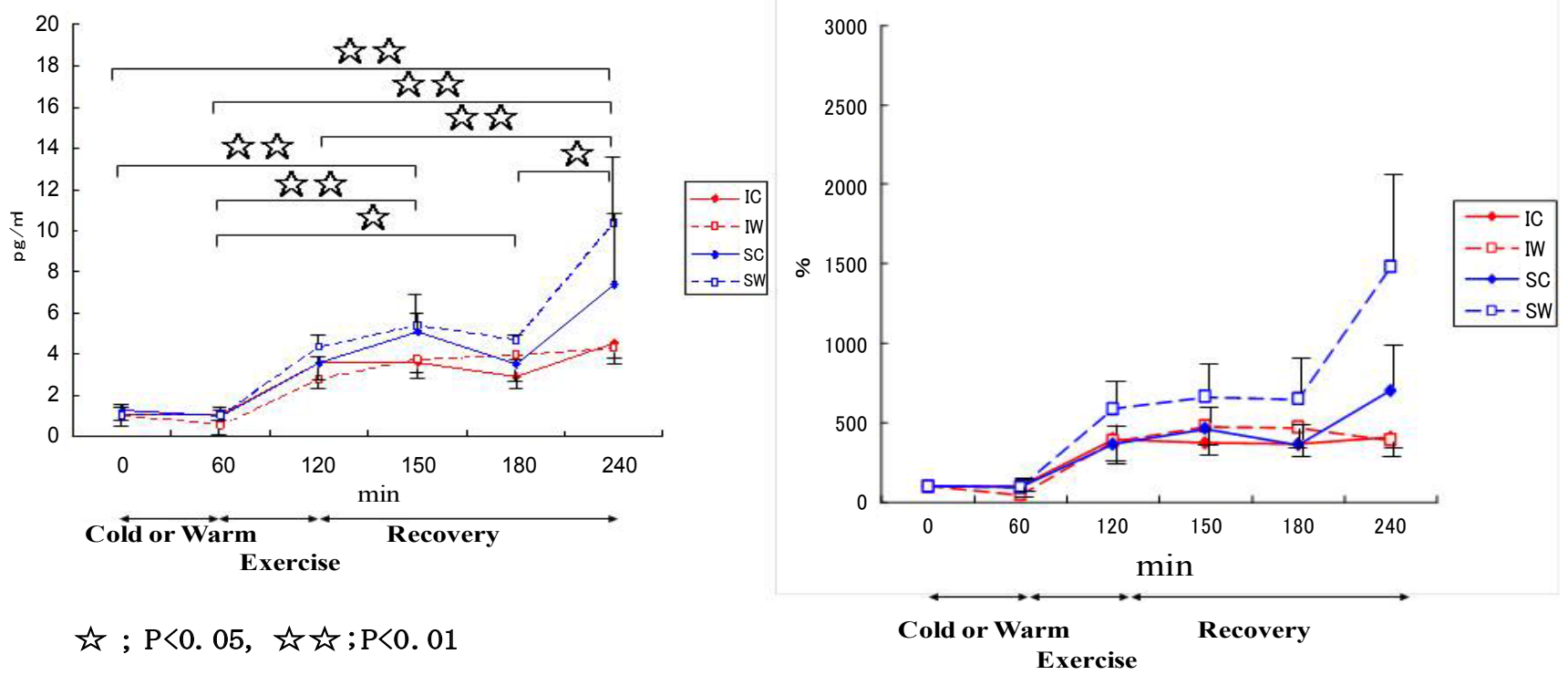

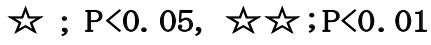

Figure 11: Responses of IL-6 to exercise in cool and warm environments [44].

I: inline skaters; S: short-track skaters; C: cool; W: warm.
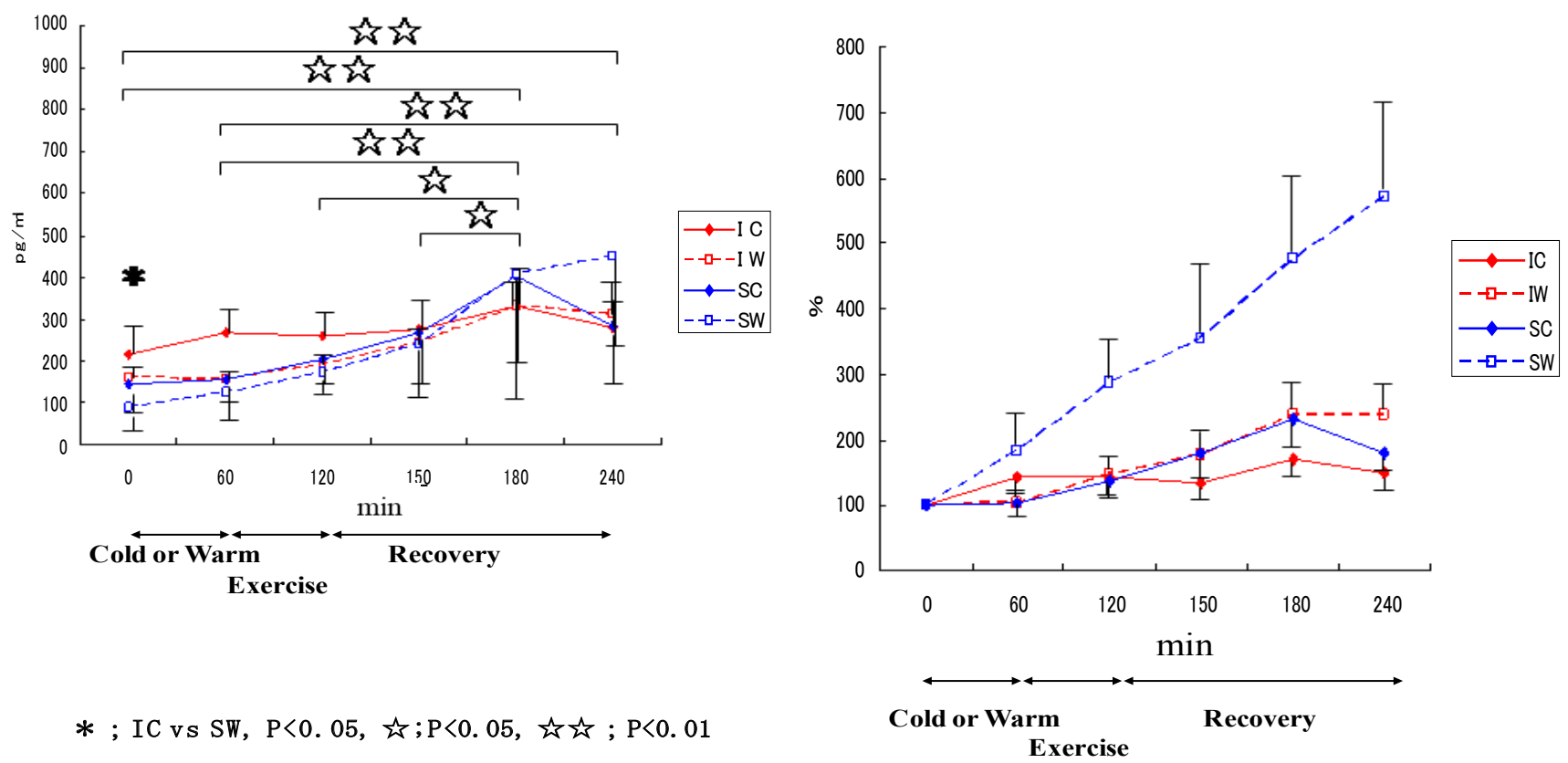

* ; IC vs SW, $\mathrm{P}<0.05$, tris $\mathrm{P}<0.05$, tro $; \mathrm{P}<0.01$

Figure 12: Responses of IL-1ra to exercise in cool and warm environments [44]. I: inline skaters; S: short-track skaters; C: cool; W: warm. 
for the muscle damage marker, the pattern of changes was different in that muscle damage was greater for the inline skaters when they exercised under unaccustomed cool conditions (Figure 15). In summary, this study demonstrated that cytokine responses to exercise depended on stress and body temperature elevation, independent of muscle damage. The short-track skaters showed marked increases in plasma cytokines in the warm, suggesting that cool environments did not cause cytokine and stress hormone responses to exercise.

Although pre-exercise cooling can effectively attenuate systemic inflammatory response to exhaustive exercise $[3,6]$, post-exercise cooling may not have significant effects [44-46]. During the day, evening exercise induces more marked IL-6 release than morning exercise [21], and it is recommended to exercise in cooler environments in the morning.

\section{Other Lifestyle Factors Affecting Cytokine Response to Exercise}

As for fluid supplementation, we conducted a study to evaluate the effectiveness of a hypotonic carbohydrate drink that is easy to drink and is rapidly absorbed. That is because fluid supplementation in combination with carbohydrate ingestion also prevents dehydration and heat stroke [3]. Male cyclists completed three cycling trials at $60 \% \mathrm{VO}_{2}$ max for $90 \mathrm{~min}$ in hot conditions [47]. Three different drinks were ingested ad libitum during 90-min cycling; isotonic drink, hypotonic drink and water as the placebo control. As for the fluid intake during 90-minute exercise, the subjects consumed
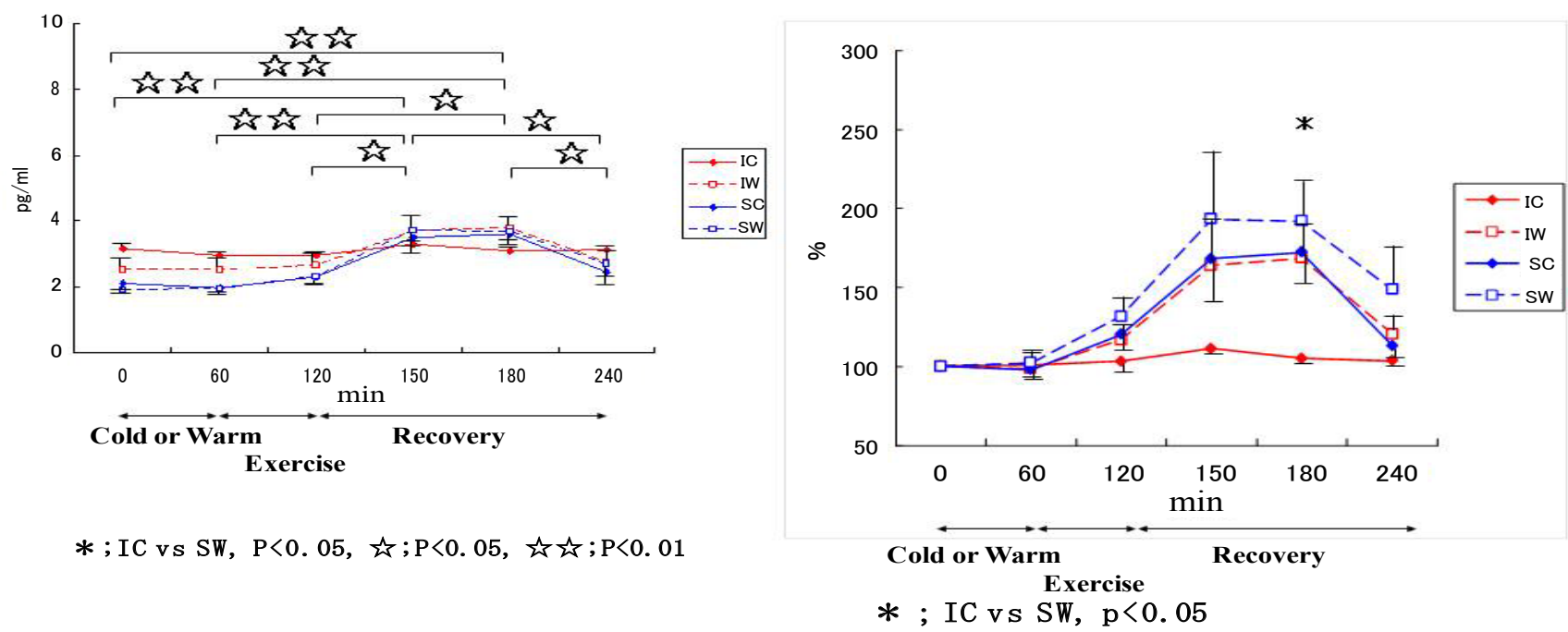

Figure 13: Responses of IL-10 to exercise in cool and warm environments [44].

I: inline skaters; S: short-track skaters; C: cool; W: warm.
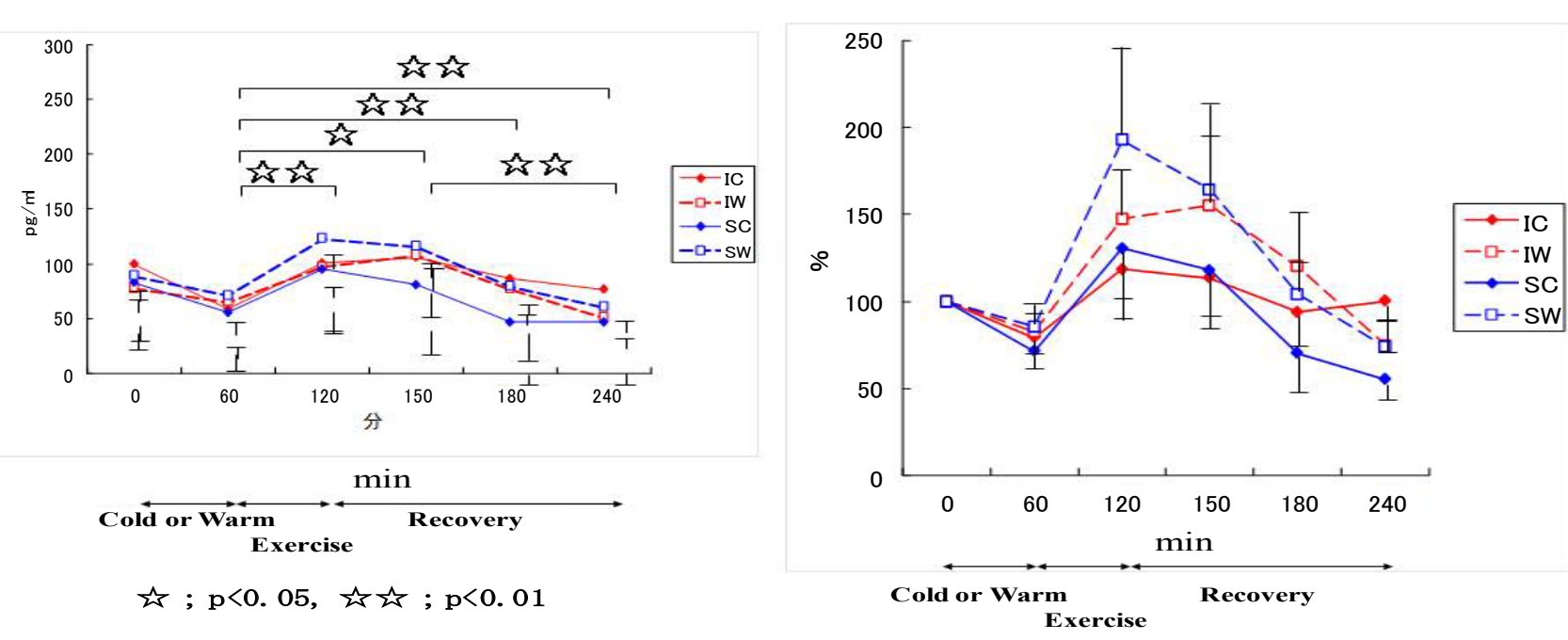

Figure 14: Responses of cortisol to exercise in cool and warm environments [44].

I: inline skaters; S: short-track skaters; C: cool; W: warm. 

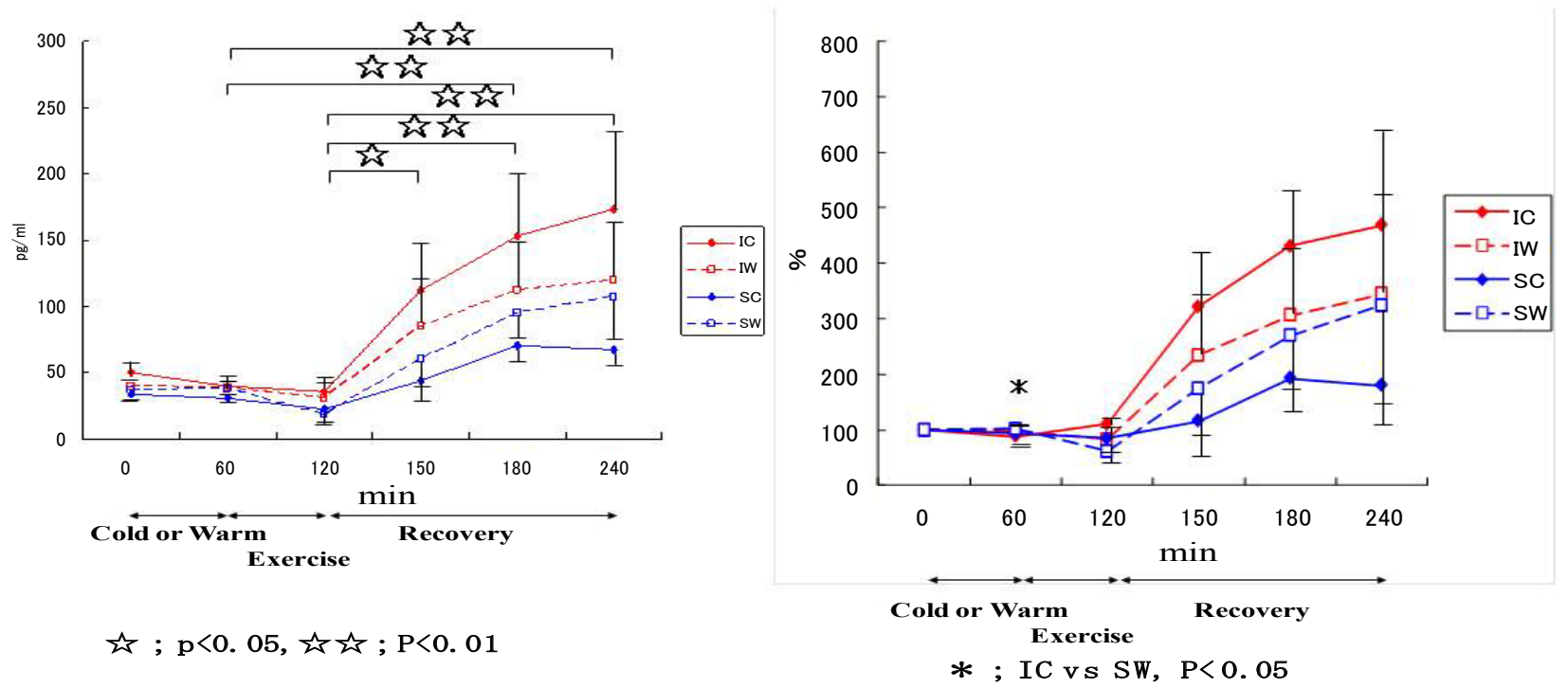

Figure 15: Responses of myoglobin to exercise in cool and warm environments [44].

I: inline skaters; S: short-track skaters; C: cool; W: warm.

more of the hypotonic drink than water. Carbohydrate drinks were effective to minimize dehydration, which was also confirmed by the plasma volume changes. As for the metabolic responses, the isotonic drink caused hyperglycemia, whereas the hypotonic drink kept the glucose level constant. Exercise induced an increase in free fatty acids, but the hypotonic drink was the most effective to spare this mobilization in the recovery period. Neutrophil responses were reduced post exercise by carbohydrate ingestion. Plasma IL- 6 response was reduced by the hypotonic drink. There were no differences in body temperature, heart rate, lactate, osmolality, muscle damage markers or cytokines other than IL-6. In summary of this drink study, carbohydrate significantly reduced free fatty acids and leukocyte responses to endurance exercise in the heat, but the isotonic glucose drink caused hyperglycemia post exercise. The hypotonic drink effectively attenuated IL-6 responses without inducing IL-1ra and IL-10 responses. These results suggest that the hypotonic drink is more appropriate to prevent dehydration and immune changes compared with the isotonic drink and water.

We have also investigated the effects of ingredients of sport drinks to prevent inflammation [41,47-49], but an appropriate regime remains to be examined at present. Especially, for women, intensive exercise in the menstruation phase of the menstruation cycle increases systemic inflammation [50], and energy and fluid supplementation during exercise must be considered depending on the phase [51]. From a nutraceutical perspective, curcumin ingestion has reduced muscle inflammation after downhill running in a mouse model of exercise-induced muscle damage and oxidative stress in humans $[16,52,53]$, but the evidence is not sufficient for other antioxidant and/or anti-inflammatory substances for the prevention of muscle damage and inflammation [54-60]. Intestinal permeability increases following exer- cise, and endotoxemia occurs, which induces systemic inflammation [3,61-63]. Therefore, other countermeasures such as intake of some functional foods for gut barrier protection, better bioavailability, and distribution [6,64], and appropriate immune responsiveness with reducing inflammation [65-67], should be examined in future studies.

\section{Conclusive Remarks}

Cytokines featured at the forefront of biomedical research in 1990s [1] and we have examined the effects of exercise on the body and their modulations together with underlying mechanisms of action. IL-6 might enhance endurance performance, but might also induce systemic inflammation, muscle damage and immunosuppression. It is possible that appropriate countermeasures such as consuming energy and fluids, and minimizing rises in body temperature during exercise, might help to maintain endurance performance without causing harmful side effects on the body resulting from inflammation.

\section{Acknowledgements}

I have conducted this work with the help of domestic and international collaborators, and gratefully appreciate them and my laboratory team for research progress. Parts of these contents were presented in the Presidential Symposium in $13^{\text {th }}$ International Society of Exercise and Immunology (ISEI) congress (Coimbra, Portugal, 2017). This work has been supported by a Grant-in-Aid for the Scientific Research (A). Also, this study was part of research activities of the Human Performance Laboratory, Organization for University Research Initiatives, Waseda University.

\section{Conflicts of Interest}

The author declares no conflicts of interest. 


\section{References}

1. Thomson AW (1998) The cytokine handbook. Third edition. Academic Press, California, USA.

2. Suzuki K, Nakaji S, Yamada M, Totsuka M, Sato K, et al. (2002) Systemic inflammatory response to exhaustive exercise. Cytokine kinetics. Exerc Immunol Rev 8: 6-48

3. Lim CL, Suzuki K (2017) Systemic inflammation mediates the effects of endotoxemia in the mechanisms of heat stroke. Biol Med (Aligarh) 9: 1000376.

4. Hung YL, Suzuki K (2017) The pattern recognition receptors and lipopolysaccharides (LPS)-induced systemic inflammation. Int J Res Studies Med Health Sci 2: 1-7.

5. Suzuki K, Nakaji S, Kurakake S, Totsuka M, Sato K, et al. (2003) Exhaustive exercise and type-1/type-2 cytokine balance with special focus on interleukin-12 p40/p70. Exerc Immunol Rev 9: 48-57.

6. Suzuki K (2018) Inflammatory response to exercise and its prevention. Current Topics in Biochemical Research 19: $37-$ 42.

7. Pedersen BK, Febbraio MA (2008) Muscle as an endocrine organ: focus on muscle-derived interleukin-6. Physiol Rev 88: 1379-1406.

8. Peake JM, Della Gatta P, Suzuki K, Nieman DC (2015) Cytokine expression and secretion by skeletal muscle cells: regulatory mechanisms and exercise effects. Exerc Immunol Rev 21: 8-25.

9. Goh JM, Lim CL, Suzuki K (2019) Effects of endurance-, strength-, and concurrent training on cytokines and inflammation. Concurrent Aerobic and Strength Training, 125-138.

10. Suzuki K (2018) Cytokine response to exercise and its modulation. Antioxidants 7: 17.

11. Suzuki K, Sato H, Kikuchi T, Abe T, Nakaji S, et al. (1996) Capacity of circulating neutrophils to produce reactive oxygen species after exhaustive exercise. J Appl Physiol 81: 1213-1222.

12. Suzuki K, Naganuma S, Totsuka M, Suzuki KJ, Mochizuki $M$, et al. (1996) Effects of exhaustive endurance exercise and its one-week daily repetition on neutrophil count and functional status in untrained men. Int J Sports Med 17: 205-212.

13. Suzuki $K$, Hasegawa $H$, Endo $T$, Sato $H$, Koie $T$, et al (1996) Effects of maximal exercise on blood leukocyte counts, cytokine levels and neutrophil chemiluminescence. J Phys Fit Nutr Immunol 6: 123-130.

14. Suzuki K, Yamada M, Kowatari K, Kudoh S, Liu Q, et al. (1998) Effects of maximal exercise on plasma and urine cytokine concentrations in athletes. J Phys Fit Nutr Immunol 8: 176-177.

15. Suzuki K (2017) Exhaustive exercise-induced neutrophilassociated tissue damage and possibility of its prevention. J Nanomedicine Biotherapeutic Discov 7: 156.

16. Suzuki K (2018) Involvement of neutrophils in exerciseinduced muscle damage. Gen Int Med Clin Innov 3: 1-8.

17. Yamada M, Suzuki K, Kudo S, Totsuka M, Nakaji S, et al. (2002) Raised plasma G-CSF and IL-6 after exercise may play a role in neutrophil mobilization into the circulation. $J$ Appl Physiol (1985) 92: 1789-1794.

18. Suzuki K, Yamada M, Kurakake S, Okamura N, Yamaya $\mathrm{K}$, et al. (2000) Circulating cytokines and hormones with immunosuppressive but neutrophil-priming potentials rise after endurance exercise in humans. Eur J Appl Physiol 81: 281-287.

19. Suzuki K, Nakaji S, Yamada M, Liu Q, Kurakake S, et al. (2003) Impact of a competitive marathon race on systemic cytokine and neutrophil responses. Med Sci Sports Exerc 35: 348-355.

20. Suzuki K, Totsuka M, Nakaji S, Yamada M, Kudoh S, et al. (1999) Endurance exercise causes interaction among stress hormones, cytokines, neutrophil dynamics, and muscle damage. J Appl Physiol 87: 1360-1367.

21. Kim, HK, Konishi M, Takahashi M, Tabata H, Endo N, et al. (2015) Effects of acute endurance exercise performed in the morning and evening on inflammatory cytokine and metabolic hormone responses. PLoS ONE 10: 0137567.

22. Ma S, Huang Q, Tominaga T, Liu C, Suzuki K (2018) An 8-Week Ketogenic Diet Alternated Interleukin-6, Ketolytic and Lipolytic Gene Expression, and Enhanced Exercise Capacity in Mice. Nutrients 10: 1696.

23. Ma S, Suzuki K (2019) Keto-adaptation and endurance exercise capacity, fatigue recovery, and exercise-induced muscle and organ damage prevention. Sports 7: 40 .

24. Suzuki K, Peake J, Nosaka K, Okutsu M, Abbiss CR, et al. (2006) Changes in markers of muscle damage, inflammation and HSP70 after an Ironman triathlon race. Eur J Appl Physiol 98: 525-534.

25. Sugama K, Suzuki K, Yoshitani K, Shiraishi K, Kometani T (2012) IL-17, neutrophil activation and muscle damage following endurance exercise. Exerc Immunol Rev 18: 116127.

26. Sugama K, Suzuki K, Yoshitani K, Shiraishi K, Kometani T (2013) Urinary excretion of cytokines versus their plasma levels after endurance exercise. Exerc Immunol Rev 19: 29-48.

27. Sugama K, Suzuki K, Yoshitani K, Shiraishi K, Miura S, et al. (2015) Changes of thioredoxin, oxidative stress markers, inflammation and muscle/renal damage following intensive endurance exercise. Exerc Immunol Rev 21: 130-142.

28. Nosaka K, Abbiss CR, Watson G, Wall B, Suzuki K, et al. (2010) Recovery following an Ironman triathlon. Eur J Sport Sci 10: 159-165.

29. MacDonald C, Wojtaszewski JF, Pedersen BK, Kiens B, Richter EA (2003) Interleukin-6 release from human skeletal muscle during exercise: relation to AMPK activity. J Appl Physiol (1985) 95: 2273-2277.

30. Peake J, Wilson G, Hordern M, Suzuki K, Yamaya K, et al. (2004) Changes in neutrophil surface receptor expression, degranulation, and respiratory burst activity after moderateand high-intensity exercise. J Appl Physiol 97: 612-618.

31. Peake JM, Suzuki K, Hordern M, Wilson G, Nosaka K, et al. (2005) Plasma cytokine changes in relation to exercise intensity and muscle damage. Eur J Appl Physiol 95: 514-521.

32. Peake JM, Suzuki K, Wilson G, Hordern M, Nosaka K, et al. (2005) Exercise-induced muscle damage, plasma cytokines, and markers of neutrophil activation. Med Sci Sports Exerc 3: 737-745.

33. Hirose L, Nosaka K, Newton M, Laveder A, Kano M, et al. (2004) Changes in inflammatory mediators following eccentric exercise of the elbow flexors. Exerc Immunol Rev 10: $75-90$.

34. Peake J, Nosaka K, Suzuki K (2005) Characterization of inflammatory responses to eccentric exercise in humans. Exerc Immunol Rev 11: 64-85. 
35. Peake J, Nosaka K, Muthalib M, Suzuki K (2006) Systemic inflammatory responses to maximal versus submaximal lengthening contractions of the elbow flexors. Exerc Immunol Rev 12: 72-85.

36. Kanda K, Sugama K, Hayashida H, Sakuma J, Kawakami Y, et al. (2013) Eccentric exercise-induced delayed-onset muscle soreness and changes in markers of muscle damage and inflammation. Exerc Immunol Rev 19: 72-85.

37. Kanda K, Sugama K, Sakuma J, Kawakami Y, Suzuki $\mathrm{K}$ (2014) Evaluation of serum leaking enzymes and investigation into new biomarkers for exercise-induced muscle damage. Exerc Immunol Rev 20: 39-54.

38. Kanda K, Sakuma J, Akimoto T, Kawakami Y, Suzuki K (2017) Detection of titin fragments in urine in response to exercise-induced muscle damage. PLoS One 12: e0181623.

39. Nieman DC, Davis JM, Henson DA, Gross SJ, Dumke CL, et al. (2005) Muscle cytokine mRNA changes after $2.5 \mathrm{~h}$ of cycling: Influence of carbohydrate. Med Sci Sports Exerc 37: 1283-1290.

40. Ross ML, Halson SL, Suzuki K, Garnham A, Hawley JA et al. (2010) Cytokine responses to carbohydrate ingestion during recovery from exercise-induced muscle injury. $J$ Interferon Cytokine Res 30: 329-337.

41. Tanisawa K, Suzuki K, Ma S, Kondo S, Okugawa S, et al. (2018) Effects of ingestion of different amounts of carbohydrate after endurance exercise on circulating cytokines and markers of neutrophil activation. Antioxidants 7: 51 .

42. Peake J, Peiffer JJ, Abbiss CR, Nosaka K, Okutsu M, et al. (2008) Body temperature and its effect on leukocyte mobilization, cytokines and markers of neutrophil activation during and after exercise. Eur J Appl Physiol 102: 391-401.

43. Peake J, Peiffer JJ, Abbiss CR, Nosaka K, Laursen PB, et al. (2008) Carbohydrate gel ingestion and immunoendocrine responses to cycling in temperate and hot conditions. Int $\mathrm{J}$ Sport Nutr Exerc Metab 18: 229-246.

44. Kondou T, Ogawa K, Terada O, Kim K, Okutsu M, et al. (2009) Cytokine response to exercise during exposure to cold. Jpn J Complement Altern Med 6: 89-95.

45. Peake JM, Roberts LA, Figueiredo VC, Egner I, Krog S, et al. (2017) The effects of cold water immersion and active recovery on inflammation and cell stress responses in human skeletal muscle after resistance exercise. J Physiol 595: 695-711.

46. Kim K, Suzuki K, Peake J, Ahn N, Ogawa K, et al. (2014) Physiological and leukocyte subset responses to exercise and cold exposure in cold-acclimatized skaters. Biol Sport 31: 39-48.

47. Suzuki $K$, Hashimoto $H$, Oh $T$, Ishijima $T$, Mitsuda $H$, et al. (2013) The effects of sports drink osmolality on fluid intake and immunoendocrine responses to cycling in hot conditions. J Nutr Sci Vitaminol 59: 206-212.

48. Suzuki K, Shiraishi K, Yoshitani K, Sugama K, Kometani T, et al. (2014) The effect of a sports drink based on highly branched cyclic dextrin on cytokine responses to exhaustive endurance exercise. J Sports Med Phys Fitness 54: 622-630.

49. Suzuki K, Takahashi M, Li CY, Lin SP, Tomari M, et al. (2015) The acute effects of green tea and carbohydrate co-ingestion on systemic inflammation and oxidative stress during sprint cycling. Appl Physiol Nutr Metabol 40: 9971003.

50. Hayashida H, Shimura M, Sugama K, Kanda K, Suzuki
$\mathrm{K}$ (2016) Exercise-induced inflammation during different phases of the menstrual cycle. Physiother Rehabil 1: 121.

51. Hashimoto H, Ishijima T, Hayashida H, Suzuki K, Higuchi M (2014) Menstrual cycle phase and carbohydrate ingestion alter immune response following endurance exercise and high intensity time trial performance test under hot conditions. J Int Soc Sports Nutr 11: 39.

52. Kawanishi N, Kato K, Takahashi M, Mizokami T, Otsuka Y, et al. (2013) Curcumin attenuates oxidative stress following downhill running-induced muscle damage. Biochem Biophys Res Commun 441: 573-578.

53. Takahashi M, Suzuki K, Kim HK, Otsuka Y, Imaizumi A, et al. (2014) Effects of curcumin supplementation on exerciseinduced oxidative stress in humans. Int J Sports Med 35: 469-475.

54. Shing CM, Peake J, Suzuki K, Okutsu M, Pereira R (2007) Effects of bovine colostrum supplementation on immune variables in highly trained cyclists. J Appl Physiol 102: 1113-1122.

55. Shing CM, Peake JM, Suzuki K, Jenkins DG, Coombes JS (2009) Bovine colostrum modulates cytokine production in human peripheral blood mononuclear cells stimulated with lipopolysaccharide and phytohemagglutinin. J Interferon Cytokine Res 29: 37-44.

56. Li CY, Suzuki K, Hung YL, Yang MS, Yu CP, et al (2017) Aloe metabolites prevent LPS-induced sepsis and inflammatory response by inhibiting mitogen-activated protein kinase activation. Am J Chin Med 45: 847-861.

57. Hung YL, Fang SH, Wang SC, Cheng WC, Liu PL, et al. (2017) Corylin protects LPS-induced sepsis and attenuates LPS-induced inflammatory response. Sci Rep 7: 46299.

58. Yada K, Suzuki K, Oginome N, Ma S, Fukuda Y, et al. (2018) Single dose administration of Taheebo polyphenol enhances endurance capacity in mice. Sci Rep 8: 14625.

59. Ma S, Yada K, Yada, K, Lee H, Fukuda Y, et al. (2017) Taheebo polyphenols attenuate FFA-induced inflammation in murine and human macrophage cell lines as inhibitor of COX-2. Front Nutr 4: 63

60. Kawamura T, Suzuki K, Takahashi M, Tomari M, Hara R, et al. (2018) Involvement of neutrophil dynamics and function in exercise-induced muscle damage and delayed onset muscle soreness: Effect of hydrogen bath. Antioxidants 7: 127.

61. Pires W, Veneroso CE, Wanner SP, Pacheco DAS, Vaz GC, et al. (2017) Association between exercise-induced hyperthermia and intestinal permeability: A systematic review. Sports Med 47: 1389-1403.

62. Van Wijck K, Lenaerts K, Grootjans J, Wijnands KA, Poeze M, et al. (2012) Physiology and pathophysiology of splanchnic hypoperfusion and intestinal injury during exercise: Strategies for evaluation and prevention. Am J Physiol Gastrointest Liver Physiol 303: 155-168.

63. Lim CL, Pyne DB, Horn P, Kalz A, Saunders P, et al. (2009) The effects of increased endurance training load on biomarkers of heat tolerance during intense exercise in the heat. Appl Physiol Nutr Metab 34: 616-624.

64. Peake JM, Suzuki K, Coombes JS (2007) The influence of antioxidant supplementation on markers of inflammation and the relationship to oxidative stress after exercise. J Nutr Biochem 18: 357-371.

65. Kawanishi N, Mizokami T, Niihara H, Yada K, Suzuki K (2016) Neutrophil depletion attenuates muscle injury after exhaustive exercise. Med Sci Sports Exerc 48: 1917-1924. 
66. Kawanishi N, Mizokami T, Niihara H, Yada K, Suzuki K (2016) Macrophage depletion by clodronate liposome attenuates muscle injury and inflammation following exhaustive exercise. Biochem Biophys Rep 5: 146-151.
67. Ma S, Suzuki K (2018) Toll-like receptor 4: Target of lipotoxicity and exercise-induced anti-inflammatory effect? Ann Nutr Food Sci 2: 1027. 